\title{
A Muscle-Specific Rehabilitation Training Method of the Human Shoulder Based on the Optimal Load Orientation Concept
}

\author{
Chao NIE ${ }^{1}$, Zhi-bin SONG ${ }^{1,{ }^{*}}$, Rong-jie KANG ${ }^{1}$ and Jian S. DAI ${ }^{1,2}$ \\ ${ }^{1}$ Key Laboratory of Mechanism Theory and Equipment Design of Ministry of Education, \\ Tianjin University, Tianjin 300072, P.R. China \\ ${ }^{2}$ King's College London, University of London, WC2R 2LS, London, United Kingdom \\ ${ }^{*}$ Corresponding author. E-mail: songzhibin@tju.edu.cn TEL: +86-022-27406136
}

Keywords: Muscle-specific rehabilitation, Musculoskeletal model, Shoulder, Optimal load orientation.

\begin{abstract}
In order to implement the high-efficiency resistance training for a specific muscle of human shoulders using the rehabilitation robots, a muscle-specific rehabilitation training method based on the optimal load orientation concept (OLOC) was proposed. A 3D mathematical musculoskeletal model of the shoulder complex was used to predict the muscle forces. In this model, 31 muscle bundles were used to represent all the muscles contributing to the shoulder function, and the Hill-type model was used to characterize the mechanical property of the muscles. The calculation results show that, for a specific muscle, there is always an optimal load orientation (OLO) of the external load which lead the activation of muscle to its maximum. Moreover, the distribution of the OLO is significantly consistent as the movement under different magnitudes of load. Thus the optimal load orientation cluster for a specific muscle, which can be used to specify a muscle-specific rehabilitation strategy, was determined. Simultaneously, the analysis suggests that the muscle-specific rehabilitation training method based on the OLOC could improve the training efficiency of specific muscles significantly.
\end{abstract}

\section{Introduction}

The shoulder complex was one of the most complicated functional regions of the human body, and it directly affected the performance of upper limb movement. Thus, a proper treatment for specific muscle was necessary for a shoulder complex with functional deficiency. Several studies indicated that rehabilitation training based on high-intensity, task-oriented and highly repetitive movements was beneficial for the restoration of shoulder function [1-7]. Therefore, the use of the robotic device became one of the most effective approaches to the rehabilitation. Rehabilitation robots could manipulate the movement of patients in several ways by applying forces to guide patients' movement in a smart way [8]. However, the rehabilitation efficiency of this method was still controversial [8-10]. In the rehabilitation with robotic devices, the training plan was normally selected from a standard options menu based on subjective clinical experience rather than objective prediction [9]. Furthermore, the training plan lacked the consideration of accurate specification about the unique characteristics of patients' physiological feature of musculoskeletal function, not to mention the muscle-specific rehabilitation.

Although the musculoskeletal movement characteristics have not been considered fully in the rehabilitation robots, researchers of biomedical engineering have done a lot of work 
focusing on the study of physiological musculoskeletal model of human shoulder. For the model of the shoulder, the typical representation of the muscles' lines of action was the line-segments model [11-14]. The models have been applied in several scenarios such as surgical simulation [13], wheelchair mechanics research $[15,16]$, neuroprostheses control $[17,18]$, etc. The direct purpose of these studies, focusing on the muscle forces, joint-contact forces and moment arms etc., was to reproduce and simulate muscle force generation patterns. To achieve this more precisely, Webb \& Blemker et al. [19] proposed a more precise and complex 3D finite element model of the shoulder to predict the moment arms and muscle forces of the shoulder. However, these musculoskeletal models based on the anatomical structure have not been applied in the rehabilitation robots adequately $[9,20]$. Therefore, in this paper, a three-dimensional mathematical musculoskeletal model [12,21,22] of the shoulder complex was used to represent the relationship of the muscles and external loads in determining the training strategy for the muscle-specific rehabilitation.

When training with the active-resisted mode of the rehabilitation robots, the robots delivered resistance against movements actively executed by the patient. The main aim of this study was to propose a muscle-specific rehabilitation training method based on the optimal load orientation concept (OLOC). Through controlling the magnitude and orientation of external loads, we could implement a high-efficiency resistance training for a specific muscle in the scenario of rehabilitation with robots' active-resisted mode. Furthermore, this paper assessed the goodness of the shoulder movement trajectory where the OLOC was adopted. This paper was organized in four sections as follows: Introduction; Methods; Results; Conclusions.

\section{Methods}

Assuming utilization of a robot to carry out the active-resisted rehabilitation training for an impaired shoulder, the robots delivered resistance against the movements executed actively by the shoulder. For a given size of load, different load direction would lead to different muscle forces, thus the specific-muscle would have different levels of muscle force. Therefore, for a specific muscle, the level of muscle force could be designed by controlling the external load.

\section{Musculoskeletal Model of Shoulder Complex}

To make the active force strategy for a specific muscle's rehabilitation, an accurate specification of musculoskeletal geometry would be necessary. Therefore, a three-dimensional mathematical musculoskeletal shoulder model[12,21,22] was used to represent the geometry structure of the skeleton and muscles of the shoulder and calculate the muscle activate state under a specific external load. The geometric parameters of the model were developed on the basis of the CT images of bones and muscles collected from the Visible Human Project (VHP) database [21]. Considering translations were negligible compared with rotations, all the articulations of the shoulder, including the sternoclavicular articulation (SC), acromiocl-avicular articulation (AC), glenohumeral articulation $(\mathrm{GH})$ and scapulathoracic articulation (ST), except for the ST articulation were assumed to be the ball-and-socket joints. However, due to the compliance of the surrounding muscles, the ST articulation was considered as a joint which allowed the scapula's translation and rotator movement with respect to the thorax. Besides, the thorax was represented as an ellipsoid. In this paper, we assumed that the upper arm and forearm moved without relative movement. Therefore, total 31 muscle bundles were used to represent the muscles that contributed to the function of the 
shoulder (see in Table 1). The muscle path was determined using the obstacle-set method proposed by Garner and Pandy [22], so that the muscle lines of action could be determined.

In this paper, the 3-element Hill-type model which was widely applied in muscle-driven simulations were used [23-26]. In this model, the muscle's driven properties was represented by four key parameters, including the tendon slack length $\left(L_{s}^{T}\right)$, pennation angle $(\beta)$, optimal muscle-fiber length $\left(L_{o}^{M}\right)$ and peak isometric muscle force $\left(F_{o}^{M}\right)$. The values of this musculotendon parameters of all the 31 muscle bundles contributing to the shoulder movement were obtained from the study reported in $[13,26,27]$. Table 1 showed those parameters where the PCSA represented the physiological cross-sectional area.

Table 1. Assumed musculotendon parameters of all the 31 muscle bundles

\begin{tabular}{|c|c|c|c|c|c|c|}
\hline Muscle bundles & Abbr. & $\begin{array}{l}\text { PCSA } \\
\left(\mathrm{cm}^{2}\right)\end{array}$ & $\begin{array}{c}L_{o}^{M} \\
(\mathrm{~cm})\end{array}$ & $\begin{array}{l}L_{s}^{T} \\
(\mathrm{~cm})\end{array}$ & $\begin{array}{l}F_{o}^{M} \\
(\mathrm{~N})\end{array}$ & $\begin{array}{c}\beta \\
(\operatorname{deg})\end{array}$ \\
\hline Subclavius & SBCL & 4.36 & 2.02 & 5.07 & 144.02 & 0 \\
\hline Serratus anterior superior & SRAs & 8.12 & 11.35 & 0.27 & 268.05 & 0 \\
\hline Serratus anterior middle & SRAm & 4.00 & 17.91 & 0.75 & 132.12 & 0 \\
\hline Serratus anterior inferior & SRAi & 8.41 & 23.15 & 0.01 & 277.51 & 0 \\
\hline Trapezius from cervical v1-6 & TRPc & 6.24 & 18.62 & 0.48 & 205.95 & 0 \\
\hline Trapezius cervical v7 & TRPc7 & 3.61 & 21.44 & 0.60 & 119.25 & 0 \\
\hline Trapezius thoracic $\mathrm{v} 1$ & TRPt1 & 3.45 & 19.37 & 0.32 & 114.01 & 0 \\
\hline Trapezius from thoracic $v 2-7$ & $\mathrm{TRPt}$ & 12.40 & 15.91 & 0.42 & 409.23 & 0 \\
\hline Levator scapulae & LVS & 3.78 & 19.02 & 0.90 & 124.78 & 0 \\
\hline Rhomboid minor & $\mathrm{RMN}$ & 6.71 & 17.55 & 0.44 & 221.51 & 0 \\
\hline Rhomboid major thoracic v1-2 & RMJt2 & 4.14 & 17.47 & 0.67 & 136.48 & 0 \\
\hline Rhomboid major thoracic v3- 4 & RMJt3 & 2.48 & 18.33 & 0.24 & 81.93 & 0 \\
\hline Pectoralis minor & PMN & 4.87 & 15.03 & 0.01 & 160.55 & 0 \\
\hline Pectoralis major clavicular & PMJc & 10.38 & 22.65 & 0.45 & 342.46 & 0 \\
\hline Pectoralis major sternal & PMJs & 14.68 & 16.58 & 9.03 & 484.35 & 0 \\
\hline Pectoralis major ribs & $\mathrm{PMJr}$ & 11.14 & 117.76 & 9.58 & 367.78 & 0 \\
\hline Latissimus dorsi thoracic & LTDt & 5.26 & 34.87 & 14.75 & 173.43 & 0 \\
\hline Latissimus dorsi lumbar & LTD1 & 12.40 & 15.91 & 0.42 & 409.23 & 0 \\
\hline Latissimus dorsi iliac & LTDi & 3.80 & 48.17 & 10.89 & 125.52 & 0 \\
\hline Deltoid clavicular & DLTc & 8.41 & 14.68 & 1.64 & 277.48 & 0 \\
\hline Deltoid acromial & DLTa & 56.38 & 6.69 & 8.56 & 1860.52 & 0 \\
\hline Deltoid scapular & DLTs & 17.19 & 17.02 & 5.93 & 567.15 & 0 \\
\hline Supraspinatus & SUPR & 20.84 & 4.28 & 13.03 & 687.84 & 0 \\
\hline Infraspinatus & INFR & 33.32 & 6.76 & 5.58 & 1099.61 & 0 \\
\hline Subscapularis & SBSC & 35.69 & 8.92 & 4.94 & 1177.93 & 0 \\
\hline Teres minor & TMN & 6.77 & 5.72 & 4.55 & 223.35 & 0 \\
\hline Teres major & TMJ & 15.59 & 14.84 & 5.79 & 514.51 & 0 \\
\hline Coracobrachialis & CRCB & 4.55 & 17.60 & 4.23 & 150.05 & 0 \\
\hline Triceps brachii long head & TRClg & 40.52 & 15.24 & 19.05 & 629.21 & 15 \\
\hline Biceps brachii short head & $\overline{\mathrm{BICs}}$ & 13.99 & 13.07 & 22.98 & 461.76 & 10 \\
\hline Biceps brachii long head & $\mathrm{BICl}$ & 11.91 & 15.36 & 22.93 & 392.91 & 10 \\
\hline
\end{tabular}




\section{Muscle Activations Prediction}

A multi-solution static equilibrium problem was solved to calculate the muscle forces for each prescribed posture of the shoulder under a certain external load. The activation was used to describe the activate status of each muscle. Based on the studies reported by Crowninshield and Brand [28,29], the muscle forces could be solved by minimizing the sum of the squares of all muscle stresses. This objective cost function strengthened the synergistic muscle load sharing for loading scenarios.

\section{The Optimal-Load Orientation (OLO) Algorithm}

Assuming a shoulder movement under a constant-magnitude external load, the muscles will contract actively to resist the load. Thus, for a specific muscle and a certain posture of shoulder, there would always be a certain load orientation which led the specific muscle to the maximum of its activation level. This orientation of load was defined to be the optimal external-load orientation $\left(\alpha_{\text {opt }}\right)$. Fig. 1 showed the flow chat which described the calculation of the optimal load orientation.

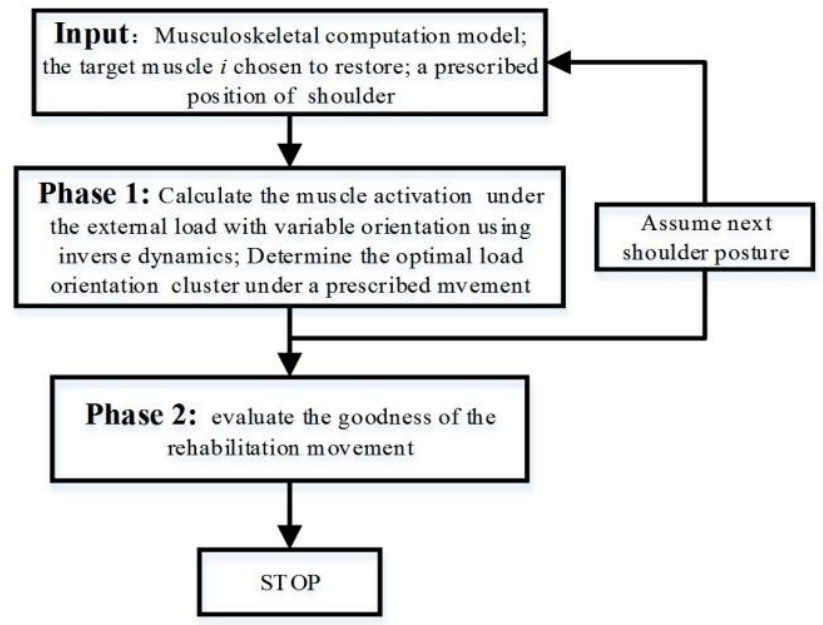

Figure 1. The flow chat of calculating the optimal load orientation and the evaluation methodology of rehabilitative movement trajectory

Only the movement of shoulder was considered, thus the upper arm and forearm were assumed to be relatively fixed. The external load was assumed to be a pure force. Fig. 2 showed the diagram of the upper limb under an external load $\left(\boldsymbol{F}_{\boldsymbol{P}}\right)$. The humerus reference frames defined in Fig. 2 were described in [21]. The $\boldsymbol{F}_{\boldsymbol{P}}$ was applied on the midpoint of the EL and EM which were the anatomical landmarks defined in [30], thus the total load was the resultant force of $\boldsymbol{F}_{\boldsymbol{P}}$ and the gravity of upper limb. During the shoulder movement, only when the direction of the external load was perpendicular to the long axis of the humerus, the muscle will suffer the greatest load effect. Therefore, the $\boldsymbol{F}_{P}$ was assumed to rotate in the normal plane perpendicular to the long axis of the humerus, and its orientation was described by the angle $\alpha$, and then different values of the activation $(x)$ were generated. Thus the $x$ - $\alpha$ curve was determined. 


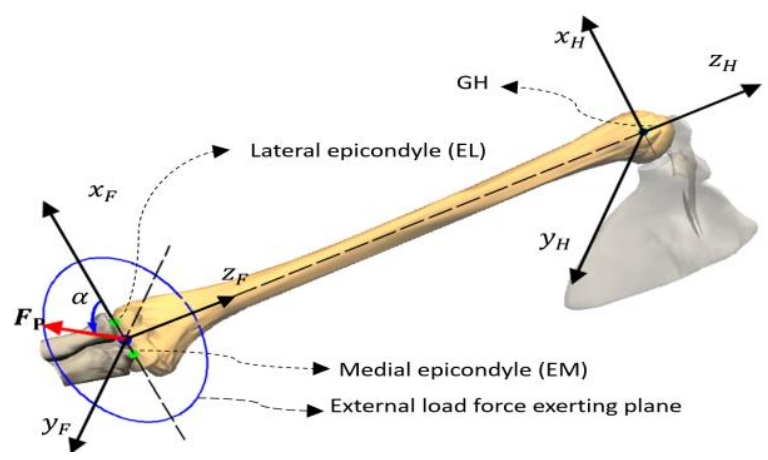

Figure 2. Schematic of external load applied to the shoulder

Fig. 3 showed the $x$ - $\alpha$ curve of deltoid clavicular part (DLTc) under the external load orientation of $2.0 \mathrm{~kg}$ (about $20 \mathrm{~N}$ ). The activations plotted in black line was the result of low-pass filtering (using a moving average filter with the span of 30). Thus the $\alpha_{\text {opt }}$ of the DLTc that led the activation to its maximum was determined. Meanwhile, the values of $\alpha_{\text {opt }}$ varied with the change of the shoulder postures (Fig. 3b).
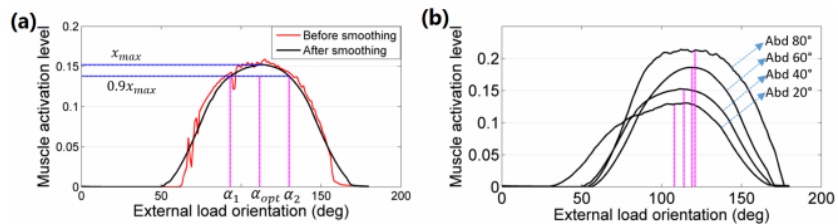

Figure 3. The $x$ - $\alpha$ curve of deltoid clavicular (DLTc) under the load of $2.0 \mathrm{~kg}$

\section{Results}

To obtain the optimal load orientation cluster and study the influence of the load magnitude, the shoulder abduction in coronal plane with the load magnitudes ranging from $0.5 \mathrm{~kg}$ to $6.0 \mathrm{~kg}$ was simulated. The differences of external-load orientation clusters under the different values of external load were quantified in terms of $R M S E$ and coefficient of determination $\left(R^{2}\right)$.

\section{The Optimal Load Orientation (OLO)}

The results of OLO demonstrated a continuity of the $x$ - $\alpha$ curves (Fig. 3), namely, the activations curve around its maximum was continuous. It meant that when the load magnitude covered in the interval $\left[\alpha_{1}, \alpha_{2}\right]$ (Fig. 3), the activation could be considered to be at a relatively high level, the same as all of the other muscles. Despite different values of optimal orientations, the results indicated a significant consistency for the activations calculated under different magnitudes of external load. Fig. 4 showed the optimal load orientation (grey circle) of deltoid scapular (DLTs) $\left(R M S E=2.62, R^{2}=0.85\right)$ obtained by regression fitting the optimal load orientations under a series of magnitudes of load. For most of the muscles, the OLO distributed in a single concentrated region (solid lines) with the different load magnitudes for abduction from 20 to $80^{\circ}$ (e.g., TRPc $\left(R M S E=4.13, R^{2}=0.85\right)$, DLTc $(R M S E=5.50$, $\left.R^{2}=0.82\right), \operatorname{DLTa}\left(R M S E=4.88, R^{2}=0.93\right), \operatorname{SUPR}\left(R M S E=3.70, R^{2}=0.78\right)$, etc. $)$. In Fig. 5 (b), the coordinate system coincided with the ground reference described in [21]. 
(a)

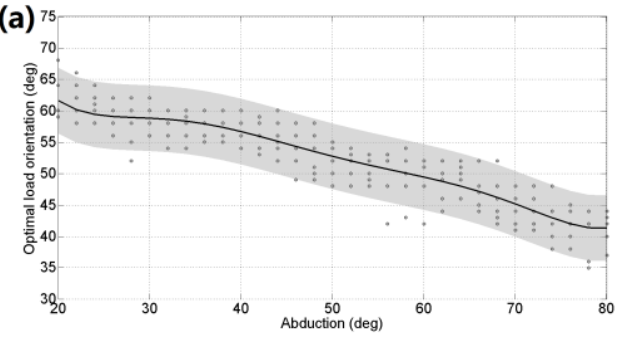

(b)

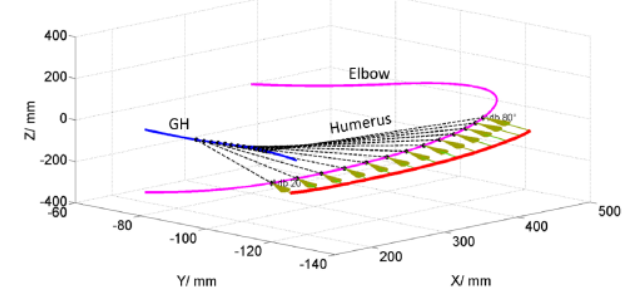

Figure 4. The optimal load orientation distribution of deltoid scapular (DLTs). (a) Single concentrated region ofDLTs. (b) The 3D representation of optimal load orientation paths. The GH center trajectory (blue line), elbow movement trajectory (pink line), virtual long axis of the humerus (black dotted line) and endpoints of external force vectors (red line) were shown vividly. The arrows represented the virtual load.

However, except for the scenarios of single region, there were also multi-region distributions for several muscles. Fig. 5 showed the optimal load orientations of teres major (TMJ). In Fig. 5, L1 (RMSE $\left.=8.44, R^{2}=0.62\right)$ and L2 $\left(R M S E=8.55, R^{2}=0.60\right)$ were the optimal orientation regions for the load sizes less than $3.0 \mathrm{~kg}$ and greater than $3.0 \mathrm{~kg}$ respectively. Therefore, for a specific muscle, the optimal load orientation path should be determined according the magnitude of the load.

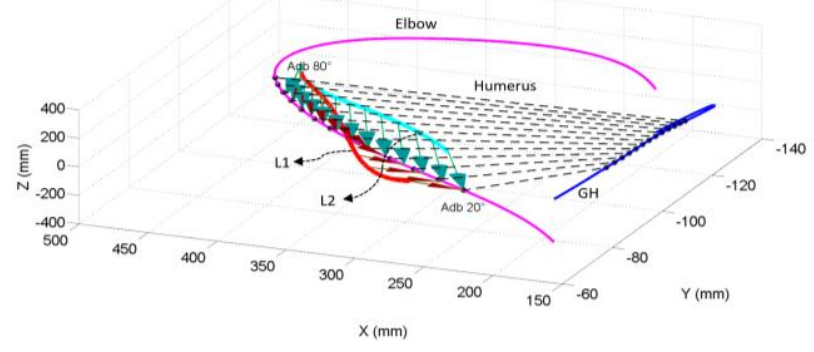

Figure 5. The Multi-region distribution of the OLO of teres major (TMJ)

\section{Muscle Training Levels with Loads Based on OLOC}

The mean activation was used to represent the training efficiency of the specific muscle. To evaluate the effect of OLOC for the improvement of the training efficiency, the weights-lifting abduction and the abduction with loads which based on OLOC were analyzed respectively. Fig. 6 showed the comparison of mean activations for the main muscles of shoulder obtained from the simulation of these two patterns. The results suggested that the muscle-specific rehabilitation training method based on the OLOC improved the training efficiency of specific muscles significantly (activations increased by an average of $537 \%$ relatively, and mean activation of all muscles increased by $165 \%$ relatively).

There were several muscles whose activations increased most significantly, including the SRA (from 0.00 to 0.21), LTDc (from 0.02 to 0.26), TRP (0.04 to 0.37), TMJ (0.11 to 0.67), LTDi (0.17 to 0.78$)$, DLTa (0.13 to 0.57$)$, SUPR ( 0.25 to 0.97$)$. However, there also was insignificance in the improvement of activation, including the INFR (increased by $1.07 \%$ relatively), $\mathrm{BICl}(5.89 \%)$. The differences of the improvement ratios were governed by the movement trajectories used for shouler's rehabilitation. The results indicated the differences of training efficiency among different muscles for a same rehabilitation movement. 

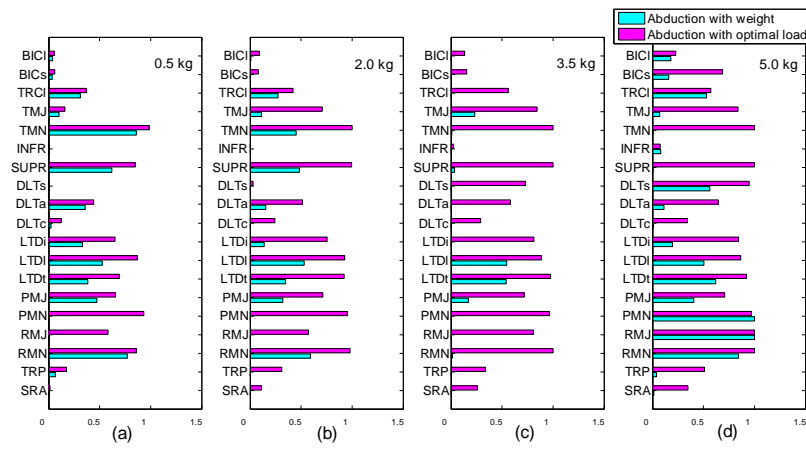

Figure 6. Comparis on of mean activations predicted by simulating weights -lifting abduction (cyan) and abduction with optimal-orientation external load (pink)

The main purpose of the training method proposed in this paper was to reduce the external load in the premise of high-efficiency training for specific muscle. However, muscles' training effects were different in response to different load magnitudes. Fig. 7 depicted the impact of the magnitudes of load. There were some muscles whose activations showed low level under the small load, but a significantly promotion with the increase of load size, such as the SRA, DLTs, TRP, BICs, etc. For these muscles, a little increasement in the load could lead to a significantly promotion in the activations of muscles. Besides, some muscles' activations changed with the magnitudes of load insignificantly, such as the PMN, LTD, DLTc, DLTa, etc. For these muscles, increasing the load had not significant effect on the promotion of training levels, thus a change of the rehabilitation movement should be considered. Therefore, when determined an external load plan for a specific muscle, an appropriate choice between increasing the level of training and reducing the value of the load must be made.

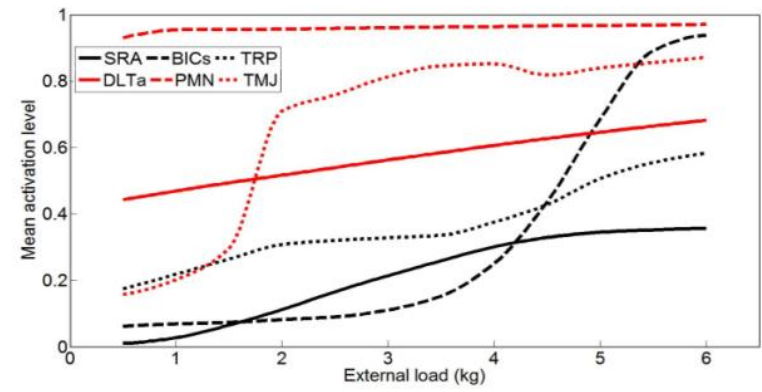

Figure 7. The impact of load magnitudes on training level for some muscles

\section{The Evaluation of Training Movement}

Assuming abduction in coronal plane with muscle-specific rehabilitation training method based on the OLOC, the mean activations and improvement ratios of different muscles were different. To evaluate the applicability of the rehabilitation movement for different muscles' training, the mean activations of the muscles were used to represent the muscle training levels. Fig. 8 showed the average activations (mean $\pm \mathrm{SD}$ ) obtained by averaging the activations' values generating under different magnitudes of the load varying from $0.5 \mathrm{~kg}$ to $6.0 \mathrm{~kg}$. On average, there were several muscles whose activations was relatively high $(>0.8)$, including the RMN ( $\mathrm{SD}=0.04), \mathrm{PMN}$ ( $\mathrm{SD}=0.01$ ), LTDt ( $\mathrm{SD}=0.08)$, LTDl ( $\mathrm{SD}=0.03)$, SUPR ( $\mathrm{SD}=0.05)$, and $\mathrm{TMN}(\mathrm{SD}=0.01)$. Thus the result indicated that the rehabilitation movement of abduction was beneficial to the training of these muscles. However, the effects of abduction in coronal plane for the training of some muscles whose activations were lower than 0.22 were ineffective, including the SRA, INFR, and BICI, especially the INFR $(x=0.15)$. Furthermore, considering 
the use of small external load in rehabilitation training generally, the SRA, INFR, and BICI may not be trained effectively.

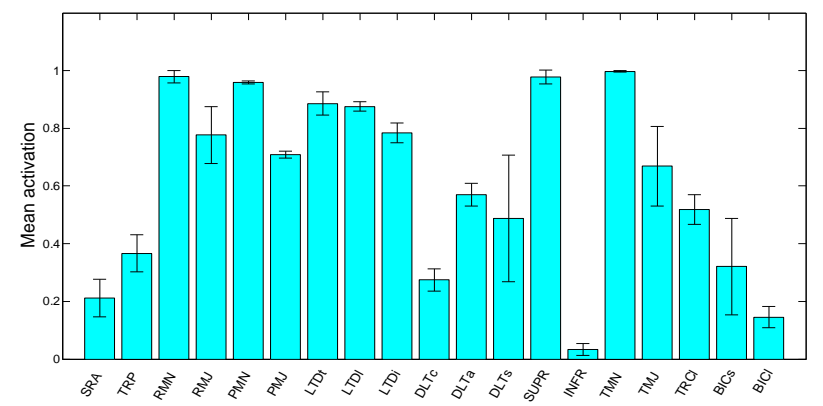

Figure 8. Average activations (mean $\pm \mathrm{SD}$ ) of the main muscles. The error bars represented the standard deviation (SD)

\section{Conclusions}

The overall aim of this study was to propose a muscle-specific rehabilitation training method for shoulder based on the optimal-load-orientation concept (OLOC). First, we calculated the optimal load orientation clusters for the mainly 31 muscle bundles of the shoulder complex, thus to achieve the high-efficiency resistance training of specific muscles through the control of external load. The analysis indicated that, for a given training movement of the shoulder, the distribution of optimal load orientation showed a significant consistency in a certain external-load magnitude interval. Secondly, after the comparison of mean activations obtained from the two movement patterns (as described in Fig. 6), it was demonstrated that the muscle-specific rehabilitation training method based on the OLOC could improve the training efficiency of the specific muscles significantly. In general, the novel aspect of this study was the development of the optimal-load-orientation concept. Once given a target muscle, the external load plan which was applied to an active-resisted rehabilitation robot was determined, thus to achieve the high-efficiency training by strengthening the training of target muscle while weakening the others.

\section{Acknowledgement}

The financial supports from the Natural Science Foundation of China (Project No. 51475322) and the Programme of Introducing Talents of Discipline to Universities ("111 Program") under Grant No. B16034 are greatly acknowledged.

\section{References}

[1] Oblak J., I. Cikajlo and Z. Matjačić. Universal haptic drive: A robot for arm and wrist rehabilitation. Neural Systems and Rehabilitation Engineering. 18 (2010) 293-302.

[2] Barreca S., S. L. Wolf, S. Fasoli and R. Bohannon. Treatment interventions for the paretic upper limb of stroke survivors: a critical review. Neurorehabilitation \& Neural Repair. 17 (2003) 220-226.

[3] HM F., D. W. WJ, S. BE, C. S. GA, S. R, V. LE, P. KD and V. H. GA. Effect of a therapeutic intervention for the hemiplegic upper limb in the acute phase after stroke: a 
single-blind, randomized, controlled multicenter trial. Stroke; a journal of cerebral circulation. 29 (1998) 785-792.

[4] Colombo R., F. Pisano, S. Micera, A. Mazzone, C. Delconte, C. M. Carrozza, P. Dario and G. Minuco. Robotic techniques for upper limb evaluation and rehabilitation of stroke patients. Neural Systems and Rehabilitation Engineering. 13 (2005) 311-324.

[5] Riener R., T. Nef and G. Colombo. Robot-aided neurorehabilitation of the upper extremities. Medical \& Biological Engineering \& Computing. 43 (2005) 2-10.

[6] Reinkensmeyer D. J., J. L. Emken and S. C. Cramer. ROBOTICS, MOTOR LEARNING, AND NEUROLOGIC RECOVERY. Annual Review of Biomedical Engineering. 6 (2004) 497-525.

[7] LumP. S., C. G. Burgar, P. C. Shor, M. Majmundar and M. V. D. Loos. Robot-assisted movement training compared with conventional therapy techniques for the rehabilitation of upper-limb motor function after stroke. Archives of Physical Medicine \& Rehabilitation. 83 (2002) 952-959.

[8] Prange G. B. Rehabilitation robotics: stimulating restoration of arm function after stroke. Enschede, the Netherlands, 2009.

[9] Fregly B. J., M. L. Boninger and D. J. Reinkensmeyer. Personalized neuromusculoskeletal modeling to improve treatment of mobility impairments: a perspective from European research sites. Journal of Neuroengineering \& Rehabilitation. 9 (2011) 1-11.

[10] Vegter R. J. K., J. Hartog, S. D. Groot, C. J. Lamoth, M. J. Bekker, J. W. V. D. Scheer, L. H. V. V. D. Woude and D. H. E. J. Veeger. Early motor learning changes in upper-limb dynamics and shoulder complex loading during handrim wheelchair propulsion. Journal of Neuroengineering \& Rehabilitation 12 (2015) 1-14.

[11] Fc V. D. H. A finite element musculoskeletal model of the shoulder mechanism. Journal of Biomechanics 27 (1994) 551-569.

[12] GARNER B. A. and M. G. PANDY. Musculoskeletal model of the upper limb based on the visible human male dataset. Computer Methods in Biomechanics \& Biomedical Engineering. 4 (2001) 93-126.

[13] Holzbaur K. R. S., W. M. Murray and S. L. Delp. A Model of the Upper Extremity for Simulating Musculoskeletal Surgery and Analyzing Neuromuscular Control. Annals of Biomedical Engineering. 33 (2005) 829-840.

[14] Nikooyan A. A., H. E. J. Veeger, E. K. J. Chadwick, M. Praagman and F. C. T. V. D. Helm. Development of a comprehensive musculoskeletal model of the shoulder and elbow. Medical \& Biological Engineering \& Computing. 49 (2011) 1425-1435.

[15] Helm F. C. T. V. D. and H. E. J. Veeger. Quasi-static analysis of muscle forces in the shoulder mechanism during wheelchair propulsion. Journal of Biomechanics. 29 (1996) 39-52.

[16] Woude L. H. V. V. D., H. E. J. Veeger, A. J. Dallmeijer, T. W. J. Janssen and L. A. Rozendaal. Biomechanics and physiology in active manual wheelchair propulsion 1. Medical Engineering \& Physics. 23 (2002) 713-733. 
[17] Hincapie J. G., D. Blana, E. K. Chadwick and R. F. Kirsch. Musculoskeletal model-guided, customizable selection of shoulder and elbow muscles for a C5 SCI neuroprosthesis. Neural Systems and Rehabilitation Engineering. 16 (2008) 255-263.

[18] Blana D., R. F. Kirsch and E. K. Chadwick. Combined feedforward and feedback control of a redundant, nonlinear, dynamic musculoskeletal system. Medical \& Biological Engineering. 47 (2009) 533-542.

[19] Webb J. D., S. S. Blemker and S. L. Delp. 3D finite element models of shoulder muscles for computing lines of actions and moment arms. Computer Methods in Biomechanics \& Biomedical Engineering. 17 (2012) 829-837.

[20] Zhang F., W. Xiangyu, Y. Fu and S. K. Agrawal. A human-robot interaction modeling approach for hand rehabilitation exoskeleton using biomechanical technique. In: Intelligent Robots and Systems (IROS), 2015 IEEE/RSJ International Conference on. 2015, pp. 5593-5598.

[21] GARNER B. A. and M. G. PANDY. A Kinematic Model of the Upper Limb Based on the Visible Human Project (VHP) Image Dataset. Computer Methods in Biomechanics \& Biomedical Engineering. 2 (1999) 107-124.

[22] GARNER B. A. and M. G. PANDY. The Obstacle-Set Method for Representing Muscle Paths in Musculoskeletal Models. Computer Methods in Biomechanics \& Biomedical Engineering. 3 (2000) 1-30.

[23] Zajac F. E. Muscle and tendon: properties, models, scaling, and application to biomechanics and motor control. Critical Reviews in Biomedical Engineering. 17 (1988) 359-411.

[24] Epstein M. and W. Herzog. Theoretical models of skeletal muscle: biological and mathematical considerations. Wiley, 1998.

[25] Winters J. M. and L. Stark. Muscle models: What is gained and what is lost by varying model complexity. Biological Cybernetics. 55 (1987) 403-420.

[26] Millard M., T. Uchida, A. Seth and S. L. Delp. Flexing computational muscle: modeling and simulation of musculotendon dynamics. Journal of Biomechanical Engineering. 135 (2013) 021005.

[27] Garner B. A. and M. G. Pandy. Estimation of Musculotendon Properties in the Human Upper Limb. Annals of Biomedical Engineering 31 (2003) 207-220.

[28] Crowninshield R. D. and R. A. Brand. A physiologically based criterion of muscle force prediction in locomotion. Journal of Biomechanics. 14 (1981) 793-801.

[29] Yanagawa T., C. J. Goodwin, K. B. Shelburne, J. E. Giphart, M. R. Torry and M. G. Pandy. Contributions of the individual muscles of the shoulder to glenohumeral joint stability during abduction. Journal of Biomechanical Engineering. 130 (2008) 237-247.

[30] Wu G., F. C. T. V. D. Helm, H. E. J. Veeger, M. Makhsous, P. V. Roy, C. Anglin, J. Nagels, A. R. Karduna, K. Mcquade and X. Wang. ISB recommendation on definitions of joint coordinate systems of various joints for the reporting of human joint motion-Part II: shoulder, elbow, wrist and hand. Journal of Biomechanics. 38 (2005) 981-992. 